\title{
CT-derived pulmonary vascular metrics and clinical outcome in COVID-19 patients
}

\author{
Pietro Spagnolo ${ }^{1 \# \wedge}$, Andrea Cozzi ${ }^{2 \#}$, Riccardo Alessandro Foà ${ }^{3 \wedge}$, Angelo Spinazzola $^{3}$, \\ Lorenzo Monfardini ${ }^{4} \wedge$, Claudio Bnà ${ }^{4}$, Marco Ali $^{1,5} \wedge$, Simone Schiaffino ${ }^{1 \wedge}$, Francesco Sardanelli ${ }^{1,2} \wedge$ \\ ${ }^{1}$ Unit of Radiology, IRCCS Policlinico San Donato, San Donato Milanese, Italy; ${ }^{2}$ Department of Biomedical Sciences for Health, Università degli \\ Studi di Milano, Milano, Italy; ${ }^{3}$ Unit of Interventional Radiology, Department of Radiology, ASST Crema - Ospedale Maggiore, Crema, Italy; ${ }^{4}$ Unit \\ of Interventional Radiology, Unit of Radiology, Fondazione Poliambulanza Istituto Ospedaliero, Brescia, Italy; ${ }^{5}$ Unit of Diagnostic Imaging and \\ Stereotactic Radiosurgery, C.D.I. Centro Diagnostico Italiano S.p.A., Milano, Italy \\ \# These authors contributed equally to this work.
}

Correspondence to: Simone Schiaffino. Unit of Radiology, IRCCS Policlinico San Donato, Via Rodolfo Morandi 30, 20097 San Donato Milanese, Italy. Email: schiaffino.simone@gmail.com.

\begin{abstract}
To assess pulmonary vascular metrics on chest CT of COVID-19 patients, and their correlation with pneumonia extent (PnE) and outcome, we analyzed COVID-19 patients with an available previous chest CT, excluding those performed for cardiovascular disease. From February 21 to March 21, 2020, of 672 suspected COVID-19 patients from two centers who underwent CT, 45 RT-PCR-positives (28 males, median age 75, IQR 66-81 years) with previous CTs performed a median 36 months before (IQR 12-72 months) were included. We assessed PnE, pulmonary artery (PA) diameter, ascending aorta (Ao) diameter, and PA/ Ao ratio. Most common presentations were fever and dyspnea (15/45) and fever alone (13/45). Outcome was available for 41/45 patients, 15/41 dead and 26/41 discharged. Ground-glass opacities (GGOs) alone were found in 29/45 patients, GGOs with consolidations in 15/45, consolidations alone in 1/45. All but one patient had bilateral pneumonia, 9/45 minimal, 22/45 mild, 9/45 moderate, and 5/45 severe PnE. PA diameter (median $31 \mathrm{~mm}$, IQR 28-33 mm) was larger than before (26 mm, IQR 25-29 mm) $(\mathrm{P}<0.001)$, PA/ Ao ratio (median 0.83, IQR 0.76-0.92) was higher than before (0.76, IQR 0.72-0.82) $(\mathrm{P}<0.001)$. Patients with adverse outcome (death) had higher PA diameter $(\mathrm{P}=0.001)$, compared to discharged ones. Only weak correlations were found between $\triangle \mathrm{PA}$ or $\triangle \mathrm{PA} / \mathrm{Ao}$ and $\mathrm{PnE}(\rho \leq 0.453, \mathrm{P} \leq 0.032)$, with $4 / 45$ cases with moderatesevere PnE and minimal increase in PA metrics. In conclusion, enlarged PA diameter was associated to death in COVID-19 patients, a finding deserving further investigation as a potential driver of therapy decisionmaking.
\end{abstract}

Keywords: Tomography, X-ray Computed; COVID-19; hypertension; pulmonary; pneumonia; viral; SARSCoV-2; patient outcome assessment

Submitted Apr 08, 2020. Accepted for publication Apr 27, 2020.

doi: $10.21037 /$ qims-20-546

View this article at: http://dx.doi.org/10.21037/qims-20-546

\footnotetext{
^, Pietro Spagnolo, ORCID: 0000-0003-0914-314X; Andrea Cozzi, ORCID: 0000-0003-4922-7065; Riccardo Alessandro, Foà ORCID: 0000-0003-2632-0531; Lorenzo Monfardini, ORCID: 0000-0002-7544-4170; Marco Alì, ORCID: 0000-0001-8156-7743; Simone Schiaffino, ORCID: 0000-0003-4243-888X; Francesco Sardanelli, ORCID: 0000-0001-6545-9427.
} 


\section{Introduction}

Pathophysiological lung changes in patients affected by the novel Coronavirus Disease (COVID-19) are easily detectable with computed tomography (CT) and mirror the possible evolution from viral pneumonia to acute respiratory distress syndrome (ARDS) (1-7). Bilateral interstitial abnormalities are initially present as subpleural groundglass opacities (GGOs), while progressive consolidations and reticular patterns can lately appear, reaching a large extent if cytokine dysregulation induces ARDS with diffuse alveolar and interstitial damage $(1,3,5)$.

Extensive lung consolidation and ARDS can alter pulmonary vasculature features (8), engendering pulmonary hypertension $(9,10)$, which in COVID-19 patients could also be caused by an overlap of these processes with increasingly-reported pulmonary arterial thrombosis (11-15). Early detection of pulmonary hypertension is therefore paramount to guide appropriate treatment (8) but remains a complex diagnostic challenge, also hampered by different and complementary shortcomings of available diagnostic techniques $(10,16)$. While COVID-19 patients frequently undergo unenhanced CT, administration of iodinated contrast agents for CT pulmonary angiography, which would facilitate the diagnosis of pulmonary arterial thrombosis, may be contraindicated in COVID-19 patients. However, unenhanced CT measurements of the ratio between maximum diameters of the pulmonary artery (PA) and the ascending aorta (Ao) PA/Ao ratio-were found to represent a helpful non-invasive option to detect pulmonary hypertension $(9,10,16,17)$. A cutoff value of $29 \mathrm{~mm}$ for PA diameter is generally accepted as highly suggestive of pulmonary hypertension $(16,18)$, while a PA/Ao ratio $>0.9$ has been found to be progressively correlated with pulmonary vascular impairment $(9,10,17)$.

This retrospective study, performed in two hospitals near to the first area put in quarantine during the COVID-19 pandemic in Italy, aimed to evaluate intra-individual variations of pulmonary vascular metrics in COVID-19 patients, comparing unenhanced CT scans performed after emergency room (ER) admission and previous CTs performed for any reason except cardiovascular disease assessment, and to correlate vascular metrics to pneumonia extent $(\mathrm{PnE})$ and clinical outcomes.

\section{Methods}

\section{Study population}

The local Ethics Committees approved this retrospective study (Ethics Committee of Brescia; protocol code NP 4154; approved on May 13, 2020).

COVID-19 patients who underwent thoracic CT at ASST Crema - Ospedale Maggiore, Crema, Italy; Center 1 and Fondazione Poliambulanza Istituto Ospedaliero, Brescia, Italy; Center 2 after ER admission from February 21 to March 21, 2020, were identified. At both institutions, during this timeframe, waiting times for reverse transcriptase-polymerase chain reaction (RT-PCR) results were too high to correctly triage the sheer number of suspected COVID-19 patients, CT being temporarily performed as a triaging test. We included in this study only patients who had subsequent confirmation of severe acute respiratory syndrome coronavirus 2 (SARS$\mathrm{CoV}-2$ ) infection by RT-PCR and had already performed another thoracic CT at the same institution for other clinical queries (except for cardiovascular disease): these previous CTs were considered as baseline examinations. Outcome data and arterial partial pressure of oxygen at admission $\left(\mathrm{PaO}_{2}-\mathrm{Adm}\right)$ were retrieved from clinical records.

\section{Image acquisition}

At Center 1, all examinations during the COVID-19 pandemic were performed on one of the two currentlyinstalled $64 \times 2$-slices CT scanners (Aquilion CXL, Toshiba/Canon Medical Systems, Ōtawara, Japan, or Revolution EVO, General Electric Healthcare, Chalfont St. Giles, UK), while all baseline examinations had been performed on the formerly-installed 16-slices CT scanner (Diamond Select Brilliance 16, Philips Healthcare, Best, The Netherlands). At Center 2, all current and baseline examinations were performed on a 16-slices CT scanner (LightSpeed RT 16, General Electric Healthcare, Chalfont St. Giles, UK). Patients' conditions were monitored during the procedure and all current scans were performed without the administration of iodinated contrast agents.

\section{Image analysis}

Two dedicated radiologists, with 7- and 20-year experience from each center, reviewed CTs from their own institution to assess pulmonary parenchyma, pulmonary vascular metrics, and Ao maximum diameter.

Lung parenchyma was assessed for the presence of the following features: GGOs and/or consolidation, crazy-paving pattern, pleural effusion, mediastinum lymphadenopathy. $\mathrm{PnE}$ was visually assessed according to the categories proposed by Bernheim et al. (2): 0\% (absent); 1-25\% (minimal); 26-50\% 

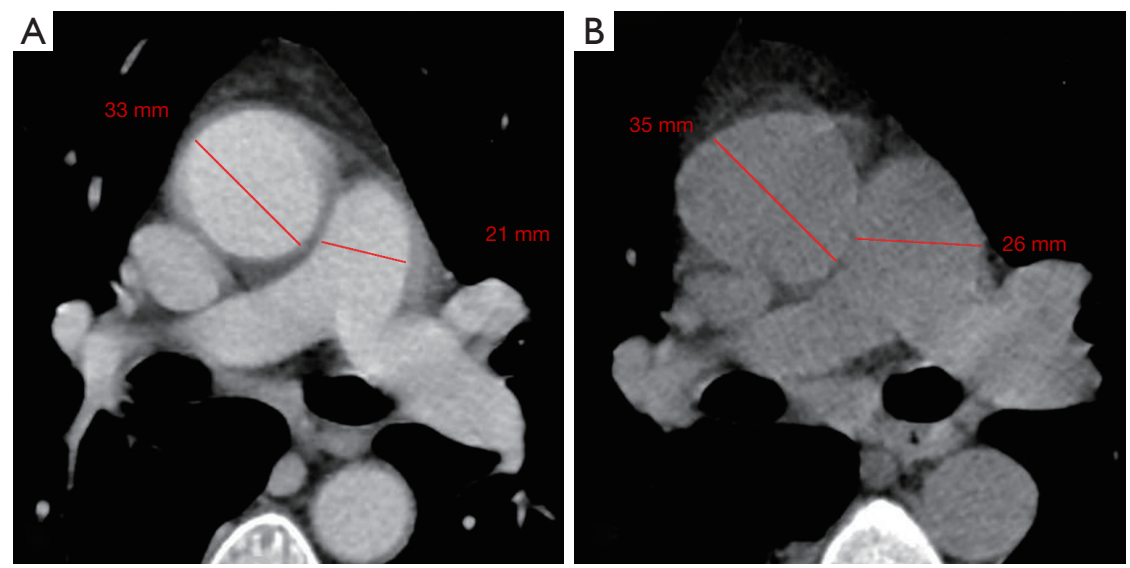

Figure 1 Pulmonary artery to ascending aorta (PA/Ao) ratio assessed on axial contrast-enhanced CT at baseline (A) and unenhanced at emergency room admission for suspected SARS-CoV-2 infection (B) in a 51-year-old woman with confirmed SARS-CoV-2 infection. Baseline CT was performed the previous year for oncologic follow-up. Soon after SARS-CoV-2 infection was confirmed, the patient began to show bilateral ground glass opacities and consolidation, with mild pneumonia extent.

(mild); 51-75\% (moderate); over 75\% (severe).

Perilesional vascular enlargement, defined as presence/ absence of enlargement of pulmonary vessels next to lung parenchymal opacities, was qualitatively assessed (4).

Finally, as described by Wells et al. (9), the PA maximum diameter at the level of its bifurcation and the Ao maximum diameter were assessed in a single slice (Figure 1), and PA/ Ao ratio then calculated.

\section{Statistical analysis}

Data were reported as mean and standard deviation (SD) or as median and IQR according to their normal or nonnormal distribution, assessed with the Shapiro-Wilk test. The Wilcoxon test was used to assess significant differences among PA/Ao ratio and PA maximum diameter before and after SARS-CoV-2 infection, Spearman's $\rho$ being used to assess correlations between vascular metrics, $\mathrm{PnE}$, admission $\mathrm{PaO}_{2}$, and hospitalization length. The Mann-Whitney $U$ test and the $\chi^{2}$ test were used to compare vascular metrics and $\mathrm{PnE}$ between groups with different outcomes.

Statistical analysis was performed using SPSS v.22.0 (IBM SPSS Inc., Chicago, IL, USA), and P values $<0.05$ were considered statistically significant.

\section{Results}

\section{Population characteristics}

In the study timeframe, 374 patients underwent triage chest CT for suspected SARS-CoV-2 infection at Center 1, 298 patients at Center 2. A previous CT examination was available for 45 of subsequently confirmed COVID-19 patients (median age 75.2 years, IQR 66.0-81.0 years), twenty-eight of them (62\%) being males. At ER admission, 15 patients (33\%) had both fever and dyspnea, $9(20 \%)$ patients had both fever and cough, 13 (29\%) only fever, $4(9 \%)$ only dyspnea, 1 (2\%) only cough, and $3(7 \%)$ respiratory insufficiency. $\mathrm{PaO}_{2}$-Adm was available for 37 patients, with a median $70 \mathrm{mmHg}$ (IQR 55-94 mmHg). Outcome data were available for $41 / 45$ patients, 15/41 (37\%) died after progression to severe ARDS and 26/41 (63\%) discharged after a median 10 days hospitalization (IQR 0-20 days).

The previous CT scans used as baseline examinations were performed a median 36 months before ER admission (IQR 12-72 months).

\section{Pulmonary parenchymal CT features}

All but one patient had bilateral pneumonia, GGOs without consolidation being found in $29(65 \%)$ patients, both GGOs and consolidation in 15 (33\%), consolidations alone in one patient only (2\%). Only one patient had associated crazypaving. PnE was categorized as minimal in nine patients (20\%), mild in $22(49 \%)$, moderate in $9(20 \%)$, and severe in $5(11 \%)$. Pulmonary perilesional vascular enlargement was found in 10 patients (22\%), 6 of them with bilateral consolidations. Five patients had bilateral pleural effusion 

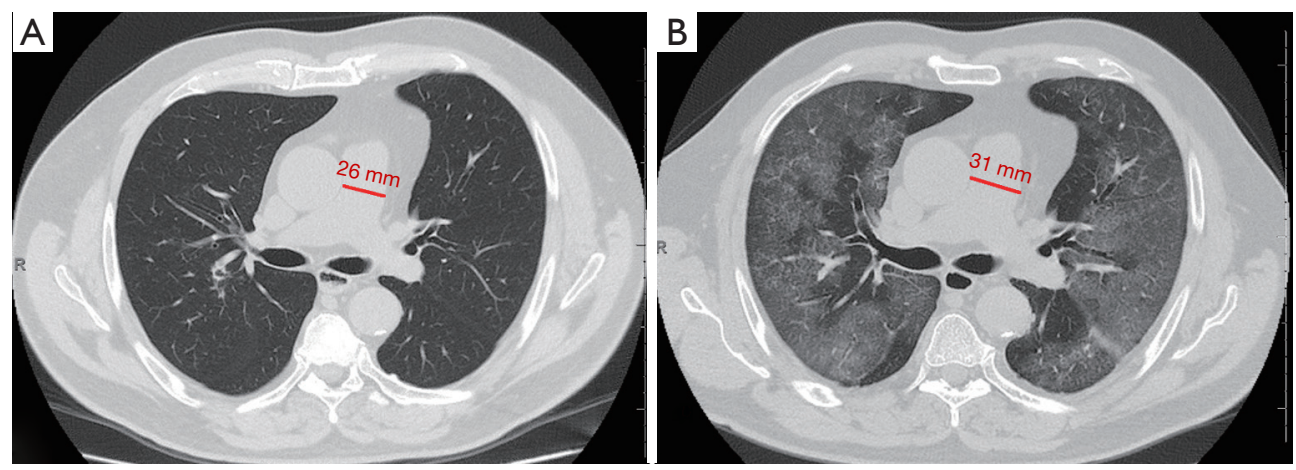

Figure 2 Axial unenhanced CT images at baseline (A) and at emergency room admission for suspected SARS-CoV-2 infection (B) in a 66-year-old man with confirmed COVID-19. Baseline CT was performed the previous year for oncologic follow-up. In (B), the patient had bilateral ground glass opacities with severe pneumonia extent and enlarged pulmonary artery.

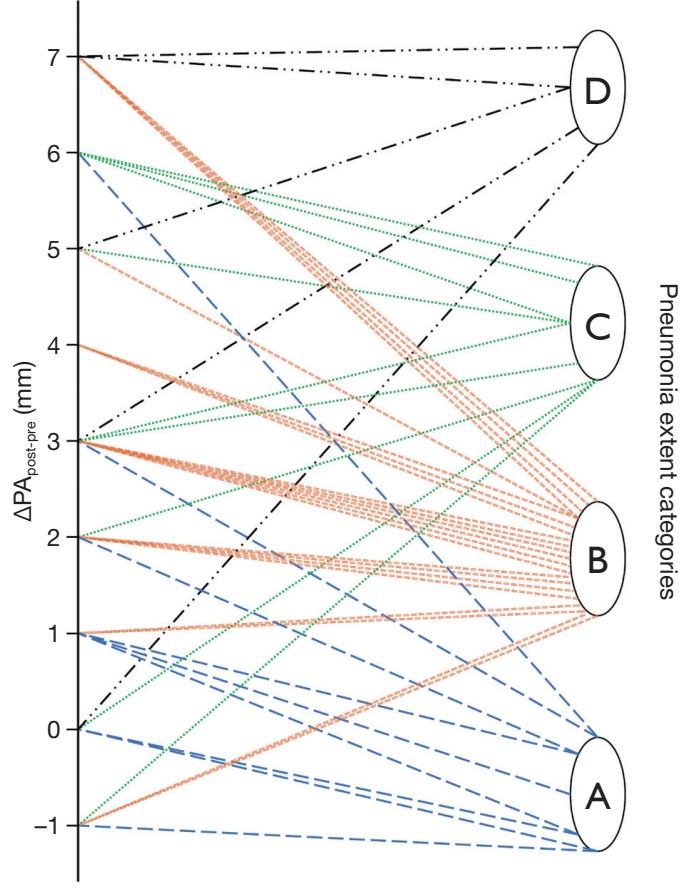

Figure $3 \Delta \mathrm{PA}_{\text {post-pre }}$ values and their relations with the four categories of pneumonia extent. A, minimal pneumonia extent; B, mild pneumonia extent; C, moderate pneumonia extent; D, severe pneumonia extent.

and three others showed mediastinum lymphadenopathies.

\section{Vascular metrics}

Median $\mathrm{PA}_{\text {pre }}$ diameter was $26 \mathrm{~mm}$ (IQR 25-29 mm), median $\mathrm{PA}_{\text {post }}$ diameter was $31 \mathrm{~mm}(\mathrm{IQR} 28-33 \mathrm{~mm})(\mathrm{P}<0.001$, Figures
1,2), and median overall $\Delta \mathrm{PA}_{\text {post-pre }}$ was $3 \mathrm{~mm}$ (IQR 1-5 mm). Median $\mathrm{Ao}_{\text {pre }}$ diameter was $35 \mathrm{~mm}(33-39 \mathrm{~mm})$, while median Ao $_{\text {post }}$ diameter was $36 \mathrm{~mm}$ (IQR 33-39 mm) (P=0.005).

Median PA/Ao ratio at ER admission (0.83, IQR 0.76$0.92)$ was significantly higher $(\mathrm{P}<0.001)$ than at baseline (0.76, IQR 0.72-0.82), the median overall value of $\triangle \mathrm{PA} /$ $\mathrm{Ao}_{\text {post-pre }}$ being 0.07 (IQR 0.02-0.11).

\section{Correlation between clinical data, radiological data, and outcome}

In patients with unfavorable outcome (i.e., death), we found lower $\mathrm{PaO}_{2}$-Adm values $(U=77, \mathrm{P}=0.023)$ and worse $\mathrm{PnE}$ $(\mathrm{P}=0.024)$ than in discharged patients. Among vascular metrics, only the $\mathrm{PA}_{\text {post }}$ diameter was significantly higher $(U=74, \mathrm{P}=0.001)$ in patients with unfavorable outcome.

As noticeable in Figure 3, $\Delta \mathrm{PA}_{\text {post-pre }}$ values showed a significant but weak correlation with $\operatorname{PnE}(\rho=0.321$, $\mathrm{P}=0.032) . \Delta \mathrm{PA} /$ Ao $_{\text {post-pre }}$ values (Figure 4 ) showed a higher correlation with $\mathrm{PnE}(\rho=0.453, \mathrm{P}=0.002)$, and a relatively higher correlation was also found between $\mathrm{PA} /$ Ao ratio at $\mathrm{ER}$ admission and $\mathrm{PnE}(\rho=0.631, \mathrm{P}<0.001)$ (Figure 5). $\mathrm{PaO}_{2}$-Adm values likewise showed a significant but intermediate correlation with $\mathrm{PnE}(\rho=-0.459, \mathrm{P}=0.004)$ and a significant but weak correlation with $\mathrm{PA} / \mathrm{Ao}$ ratio at ER admission $(\rho=-0.356, P=0.030)$. No other correlation was found among vascular metrics and the duration of hospitalization before discharge or death.

\section{Discussion}

Known disruption of pulmonary hemodynamics following 


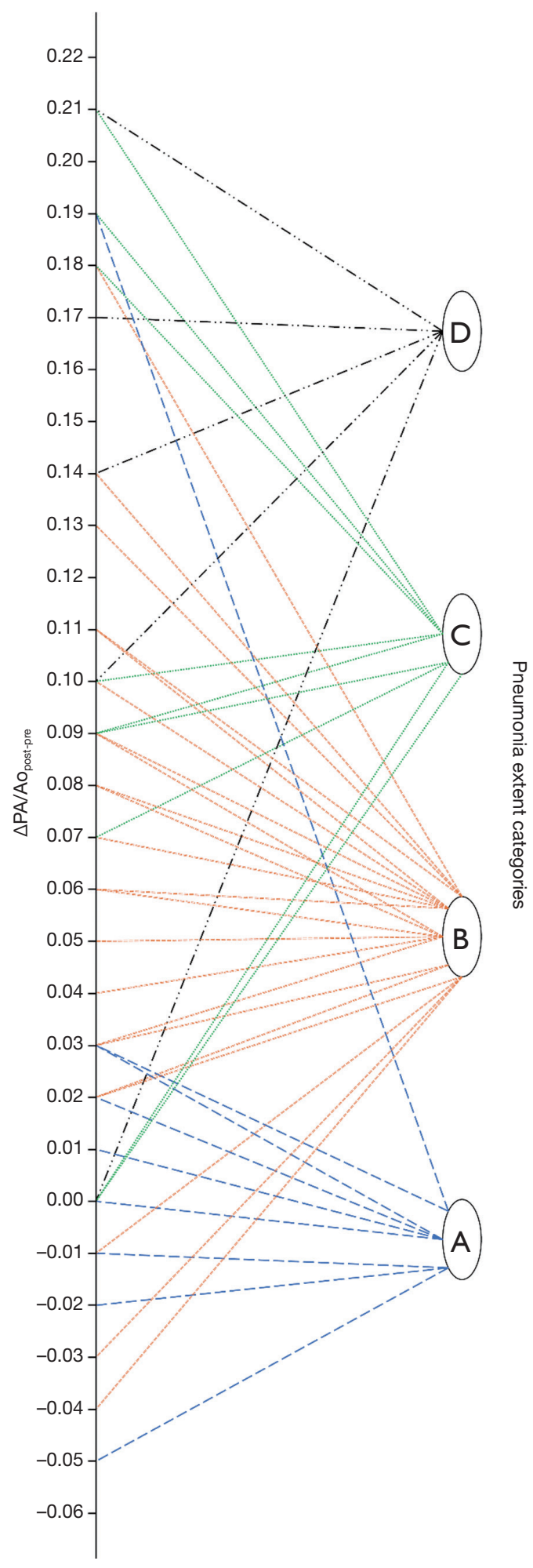

Figure $4 \Delta \mathrm{PA} / \mathrm{Ao}_{\text {post-pre }}$ values and their relations with the four categories of pneumonia extent. A, minimal pneumonia extent; B, mild pneumonia extent; C, moderate pneumonia extent; D, severe pneumonia extent.

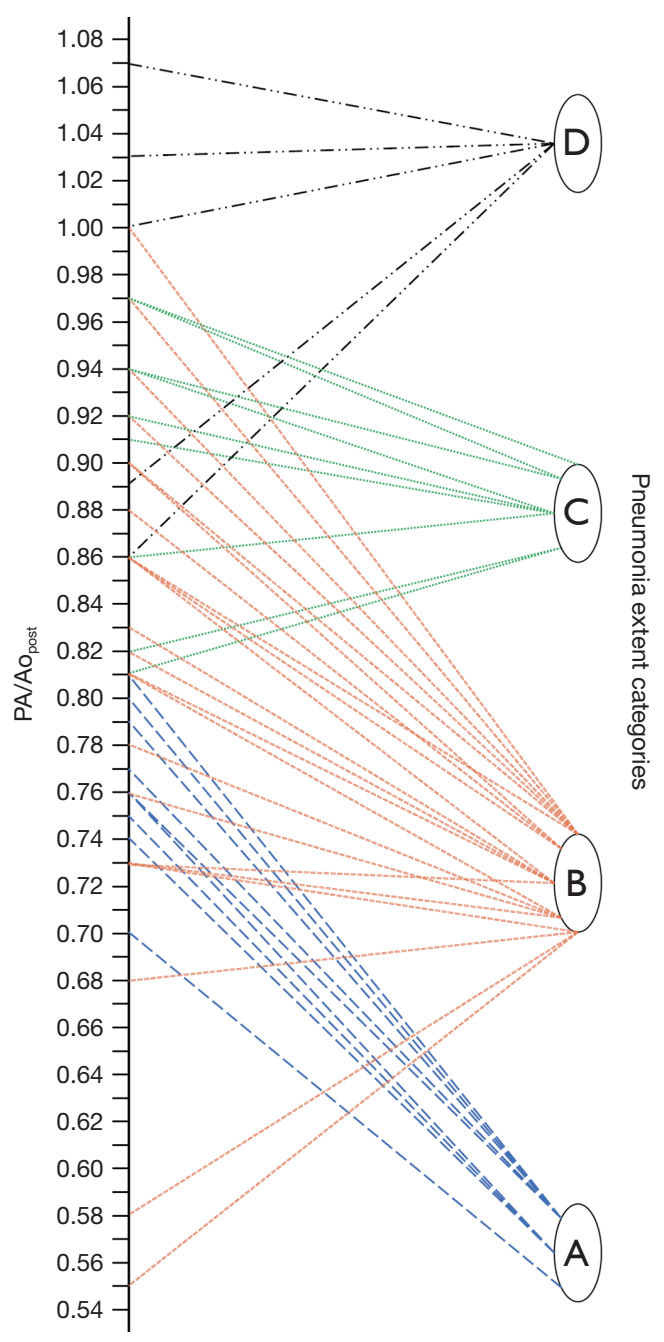

Figure $5 \mathrm{PA} / \mathrm{Ao}_{\text {post }}$ values and their relations with the four categories of pneumonia extent. A, minimal pneumonia extent; B, mild pneumonia extent; C, moderate pneumonia extent; $\mathrm{D}$, severe pneumonia extent.

large scale consolidation with inflammatory and vascular changes, expressed as vascular enlargement (4), could also be a concern in COVID-19 patients, which already present a frail intravascular equilibrium with high thromboembolic risk $(15,19-25)$. Diagnosis of pulmonary hypertension is fraught with complications $(8-10,16,17)$, but CT measurements of PA diameter and $\mathrm{PA} /$ Ao ratio constitute a helpful non-invasive option for the detection of pulmonary hypertension even in unenhanced CT, as demonstrated on large populations $(9,17)$. While such metrics are easily attainable in COVID-19 patients which already perform unenhanced CT scans and could represent a potential 


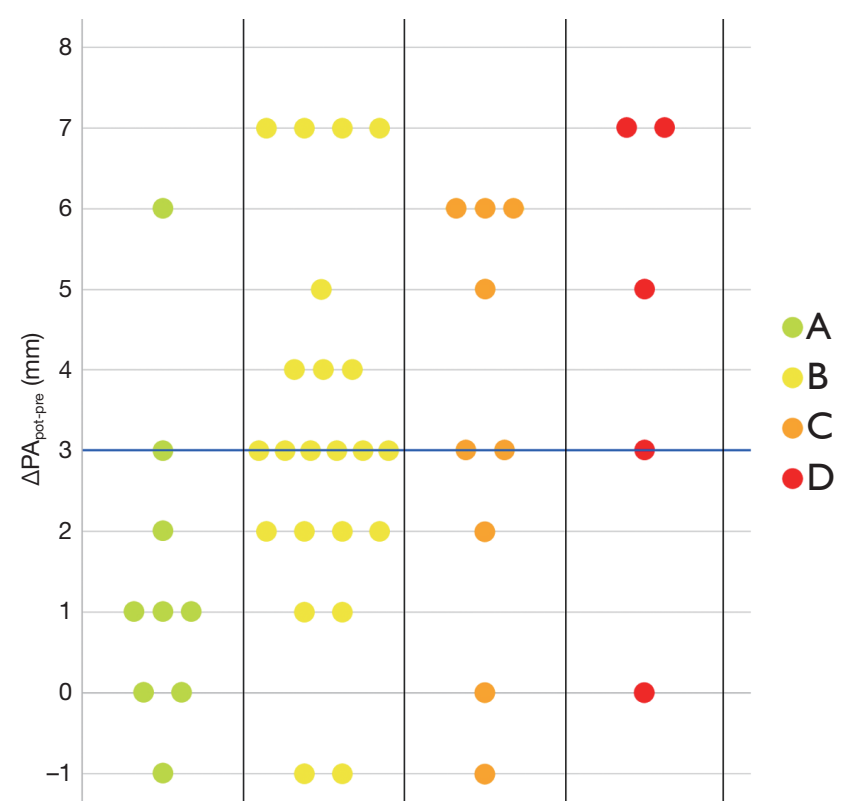

Figure 6 Category-divided scatter plot of per-patient $\Delta \mathrm{PA}_{\text {post-pre }}$ values. A, minimal pneumonia extent; $\mathrm{B}$, mild pneumonia extent; $\mathrm{C}$, moderate pneumonia extent; $\mathrm{D}$, severe pneumonia extent. The blue horizontal line represents the overall median $\Delta \mathrm{PA}_{\text {post-pre }}$ value.

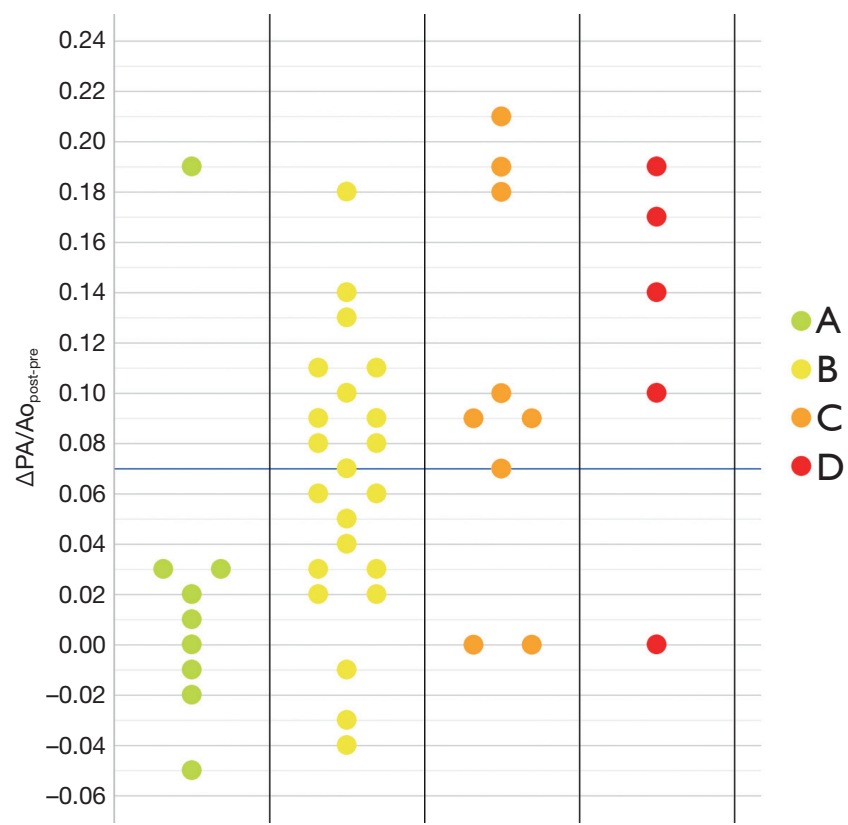

Figure 7 Category-divided scatter plot of per-patient $\triangle \mathrm{PA} / \mathrm{Ao}_{\text {post-pre }}$ values. A, minimal pneumonia extent; B, mild pneumonia extent; C, moderate pneumonia extent; $\mathrm{D}$, severe pneumonia extent. The blue horizontal line represents the overall median $\triangle \mathrm{PA} / \mathrm{Ao}_{\text {post-pre }}$ value. prognostic index, to date no study to evaluate possible pulmonary hypertension has been conducted on these patients. We therefore performed this retrospective study considering two of the first clusters of patients in the Italian COVID-19 epidemic, aiming to compare intra-patient vascular metrics on chest CT performed before and during SARS-CoV-2 infection and their correlation with baseline $\mathrm{PaO}_{2}$ and patient outcome. Including only patients with a previous CT, our data comes from fragile patients, with a median age of 75 years and main reason to perform previous examinations of oncological nature.

When measuring PA diameter after SARS-CoV-2 infection we found a median value of $31 \mathrm{~mm}$ (IQR $28-33 \mathrm{~mm}$ ), above the $27 \mathrm{~mm}$ and $29 \mathrm{~mm}$ reference values established for women and men, respectively, in the Framingham Heart Study (18). The large majority (37/45, 82\%) of COVID-19 patients from our cohort presented an increased PA caliper compared to their baseline measurements. We also found an increase both in the median PA/Ao ratio and in the PA diameter absolute median value, that rose from 26 to $31 \mathrm{~mm}$ after SARS-CoV-2 infection. Of note, all ten patients without an increased PA/Ao ratio showed already high baseline values for both components of this parameter, suggesting that an increase in pulmonary vascular pression may be related to the inflammatory status triggered by SARS-CoV-2 infection.

While an intermediate correlation was found between $\mathrm{PaO}_{2}$-Adm and $\mathrm{PnE}$, we found a weak correlation both between $\mathrm{PaO}_{2}$-Adm and $\mathrm{PA} / \mathrm{Ao}_{\text {post }}$ and between vascular metrics and PnE, as depicted in Figures 6,7. Considering the two categories with lowest $\mathrm{PnE}$, we observed 9 patients with $\triangle \mathrm{PA}_{\text {post-pre }}$ values higher than the overall median value (Figure 6), and 11 patients with $\triangle \mathrm{PA} / \mathrm{Ao}_{\text {post-pre }}$ higher than the overall median value (Figure 7). Conversely, considering the two categories with highest $\mathrm{PnE}$, we observed 4 patients with $\triangle \mathrm{PA}_{\text {post-pre }}$ values lower than the overall median value and 3 patients with $\triangle \mathrm{PA} / \mathrm{Ao}_{\text {post-pre }}$ lower than the overall median $\triangle \mathrm{PA} / \mathrm{Ao}_{\mathrm{post}}$-pre. This could be explained considering that only one of our patients had already progressed to the late phase of mostly consolidative pulmonary damage, during which-as already demonstrated in various types of acute and chronic pulmonary interstitial disease (8-10,16,17)—pulmonary hypertension severity reaches its peak. However, a relatively better correlation with $\mathrm{PnE}$ was

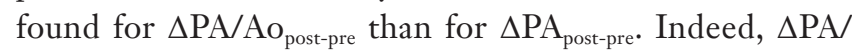
$\mathrm{Ao}_{\text {post-pre }}$ is known to be a more robust parameter for the 
evaluation of pulmonary hypertension $(10,17)$.

The overall weakness of these correlations, and especially the fact that only the $\mathrm{PA}_{\text {post }}$ diameter was higher in patients who subsequently died than in discharged patients, suggest that pulmonary vascular metrics of COVID-19 patients and their relation with outcome are also influenced by factors other than PnE. Hypotheses to explain such findings could involve both a viral-induced autoimmune endothelial damage, already associated to other coronaviruses such as SARS-CoV-1 in the early 2000s (26), and a large spectrum of procoagulant abnormalities, increasingly observed in COVID-19 patients. These abnormalities include high levels of D-dimer and fibrin degradation products, presence of antiphospholipid antibodies, longer prothrombin time and activated partial thromboplastin time $(15,19-25)$, resulting in pulmonary arterial thrombosis $(13,14)$. Patients with a low increase in pulmonary metrics could be less affected by endothelial damage and thrombosis, already reported in COVID-19 patients with poor prognosis $(12,19-25,27,28)$.

The observation that-among vascular metrics—only PA diameter was significantly higher in patients with unfavorable outcome than in discharged patients, could be related to a precocious manifestation of pulmonary arterial thrombosis. Thus, pulmonary vascular metrics on unenhanced CT scans could be useful to understand patients' conditions, possibly working as a gate-keeper to contrast-enhanced CT, especially in patients with a mismatch between $\mathrm{PnE}$ and oximeter saturation data. Early detection of pulmonary hypertension at admission could also guide treatment of COVID-19 patients, triggering modifications of anticoagulation therapies from prophylactic to therapeutic dosage $(21,27,29)$.

This study has limitations, the main one being the limited number of patients. We included all confirmed COVID-19 patients with a previous chest CT, performed for any reason (except cardiovascular diseases) at any time, even those with old previous CTs: this could bias the value of comparing previous pulmonary vascular metrics with those during infection. Moreover, tachypnoea and dyspnea are common in COVID-19 patients and motion artifacts during CT examinations could have influenced the measurements. Another limitation of our study is the manual measurement of great vessels diameters on axial scans, which is only a rough approximation for real vessels' size. A more accurate approach would be to measure vessel areas on reformatted oblique reconstructions considering vessels' axes, also considering the potential role of automatic recognition and measurement, already demonstrated for Ao on MRI (30). In addition, three-dimensional vascular metrics and flow parameters obtained by MRI or contrastenhanced CT could also be considered in future studies.

In conclusion, COVID-19 patients showed higher median PA maximum diameter and median PA/Ao ratio compared to values measured on previous chest CTs performed for any reason except cardiovascular diseases. In this limited cluster of fragile patients, PA maximum diameter showed significant difference between patients with favorable and unfavorable outcome, a finding deserving further investigation as a potential driver of therapy decision-making.

\section{Acknowledgments}

Funding: This study was partially supported by Ricerca Corrente funding from Italian Ministry of Health to IRCCS Policlinico San Donato.

\section{Footnote}

Conflicts of Interest: All authors have completed the ICMJE uniform disclosure form (available at http://dx.doi. org/10.21037/qims-20-546). SS declares to be member of speakers' bureau for General Electric and to have received travel support from Bracco. FS declares to have received grants from or to be member of speakers' bureau/advisory board for Bayer, Bracco and General Electric. The other authors have no conflicts of interest to declare.

Open Access Statement: This is an Open Access article distributed in accordance with the Creative Commons Attribution-NonCommercial-NoDerivs 4.0 International License (CC BY-NC-ND 4.0), which permits the noncommercial replication and distribution of the article with the strict proviso that no changes or edits are made and the original work is properly cited (including links to both the formal publication through the relevant DOI and the license). See: https://creativecommons.org/licenses/by-nc-nd/4.0/.

\section{References}

1. Salehi S, Abedi A, Balakrishnan S, Gholamrezanezhad A. Coronavirus Disease 2019 (COVID-19): A Systematic Review of Imaging Findings in 919 Patients. AJR Am J Roentgenol 2020. [Epub ahead of print]. doi: 10.2214/ AJR.20.23034. 
2. Bernheim A, Mei X, Huang M, Yang Y, Fayad ZA, Zhang N, Diao K, Lin B, Zhu X, Li K, Li S, Shan H, Jacobi A, Chung M. Chest CT Findings in Coronavirus Disease-19 (COVID-19): Relationship to Duration of Infection. Radiology 2020;295:200463.

3. Raptis CA, Hammer MM, Short RG, Shah A, Bhalla S, Bierhals AJ, Filev PD, Hope MD, Jeudy J, Kligerman SJ, Henry TS. Chest CT and Coronavirus Disease (COVID-19): A Critical Review of the Literature to Date. AJR Am J Roentgenol 2020. [Epub ahead of print]. doi: 10.2214/AJR.20.23202.

4. Zhao W, Zhong Z, Xie X, Yu Q, Liu J. Relation Between Chest CT Findings and Clinical Conditions of Coronavirus Disease (COVID-19) Pneumonia: A Multicenter Study. AJR Am J Roentgenol 2020. [Epub ahead of print]. doi: 10.2214/AJR.20.22976.

5. Xu R, Du M, Li L, Zhen Z, Wang H, Hu X. CT imaging of one extended family cluster of corona virus disease 2019 (COVID-19) including adolescent patients and "silent infection.” Quant Imaging Med Surg 2020;10:800-4.

6. Zhang B, Zhang J, Chen H, Yang K, Zhang S. Unmatched clinical presentation and chest CT manifestation in a patient with severe coronavirus disease 2019 (COVID-19). Quant Imaging Med Surg 2020;10:871-3.

7. Wang YXJ, Liu WH, Yang M, Chen W. The role of CT for Covid-19 patient's management remains poorly defined. Ann Transl Med 2020;8:145.

8. Beiderlinden M, Kuehl H, Boes T, Peters J. Prevalence of pulmonary hypertension associated with severe acute respiratory distress syndrome: Predictive value of computed tomography. Intensive Care Med 2006;32:852-7.

9. Wells JM, Washko GR, Han MK, Abbas N, Nath H, Mamary AJ, Regan E, Bailey WC, Martinez FJ, Westfall E, Beaty TH, Curran-Everett D, Curtis JL, Hokanson JE, Lynch DA, Make BJ, Crapo JD, Silverman EK, Bowler RP, Dransfield MT. Pulmonary Arterial Enlargement and Acute Exacerbations of COPD. N Engl J Med 2012;367:913-21.

10. Matsushita S, Matsuoka S, Yamashiro T, Fujikawa A, Yagihashi K, Kurihara Y, Nakajima Y. Pulmonary arterial enlargement in patients with acute exacerbation of interstitial pneumonia. Clin Imaging 2014;38:454-7.

11. Marongiu F, Grandone E, Barcellona D. Pulmonary thrombosis in 2019-nCoV pneumonia? J Thromb Haemost 2020. [Epub ahead of print]. doi: 10.1111/ jth.14818.

12. Dolhnikoff M, Duarte-Neto AN, de Almeida Monteiro RA, Ferraz da Silva LF, Pierre de Oliveira E, Nascimento
Saldiva PH, Mauad T, Marcia Negri E. Pathological evidence of pulmonary thrombotic phenomena in severe COVID-19. J Thromb Haemost 2020. [Epub ahead of print]. doi: $10.1111 /$ jth. 14844 .

13. Leonard-Lorant I, Delabranche X, Severac F, Helms J, Pauzet C, Collange O, Schneider F, Labani A, Bilbault P, Moliere S, Leyendecker P, Roy C, Ohana M. Acute Pulmonary Embolism in COVID-19 Patients on CT Angiography and Relationship to D-Dimer Levels. Radiology 2020. [Epub ahead of print]. doi: 10.1148/ radiol.2020201561.

14. Grillet F, Behr J, Calame P, Aubry S, Delabrousse E. Acute Pulmonary Embolism Associated with COVID-19 Pneumonia Detected by Pulmonary CT Angiography. Radiology 2020. [Epub ahead of print]. doi: 10.1148/ radiol.2020201544.

15. Xiong M, Liang X, Wei Y. Changes in Blood Coagulation in Patients with Severe Coronavirus Disease 2019 (COVID-19): a Meta-Analysis. Br J Haematol 2020. [Epub ahead of print]. doi: 10.1111/bjh.16725.

16. Lewis G, Hoey ETD, Reynolds JH, Ganeshan A, Ment J. Multi-detector CT assessment in pulmonary hypertension: techniques, systematic approach to interpretation and key findings. Quant Imaging Med Surg 2015;5:423-32.

17. Shen Y, Wan C, Tian P, Wu Y, Li X, Yang T, An J, Wang T, Chen L, Wen F. CT-Base Pulmonary Artery Measurement in the Detection of Pulmonary Hypertension. Medicine (Baltimore) 2014;93:e256.

18. Truong QA, Massaro JM, Rogers IS, Mahabadi AA, Kriegel MF, Fox CS, O’Donnell CJ, Hoffmann U.

Reference Values for Normal Pulmonary Artery Dimensions by Noncontrast Cardiac Computed Tomography. Circ Cardiovasc Imaging 2012;5:147-54.

19. Zhang $Y$, Xiao M, Zhang S, Xia P, Cao W, Jiang W, Chen H, Ding X, Zhao H, Zhang H, Wang C, Zhao J, Sun X, Tian R, Wu W, Wu D, Ma J, Chen Y, Zhang D, Xie J, Yan X, Zhou X, Liu Z, Wang J, Du B, Qin Y, Gao P, Qin X, Xu Y, Zhang W, Li T, Zhang F, Zhao Y, Li Y, Zhang S. Coagulopathy and Antiphospholipid Antibodies in Patients with Covid-19. N Engl J Med 2020. [Epub ahead of print]. doi: 10.1056/NEJMc2007575.

20. Tang N, Li D, Wang X, Sun Z. Abnormal coagulation parameters are associated with poor prognosis in patients with novel coronavirus pneumonia. J Thromb Haemost 2020;18:844-7.

21. Ranucci M, Ballotta A, Di Dedda U, Bayshnikova E, Dei Poli M, Resta M, Falco M, Albano G, Menicanti L. The procoagulant pattern of patients with COVID-19 acute 
respiratory distress syndrome. J Thromb Haemost 2020. [Epub ahead of print]. doi: 10.1111/jth.14854.

22. Han H, Yang L, Liu R, Liu F, Wu K, Li J, Liu X, Zhu C. Prominent changes in blood coagulation of patients with SARS-CoV-2 infection. Clin Chem Lab Med 2020. [Epub ahead of print]. doi: 10.1515/cclm-2020-0188.

23. Klok FA, Kruip MJHA, van der Meer NJM, Arbous MS, Gommers DAMPJ, Kant KM, Kaptein FHJ, van Paassen J, Stals MAM, Huisman MV, Endeman H. Incidence of thrombotic complications in critically ill ICU patients with COVID-19. Thromb Res 2020. [Epub ahead of print]. doi: 10.1016/j.thromres.2020.04.013.

24. Panigada M, Bottino N, Tagliabue P, Grasselli G, Novembrino C, Chantarangkul V, Pesenti A, Peyvandi F, Tripodi A. Hypercoagulability of COVID-19 patients in Intensive Care Unit. A Report of Thromboelastography Findings and other Parameters of Hemostasis. J Thromb Haemost 2020. [Epub ahead of print]. doi: 10.1111/ jth. 14850 .

25. Tan CW, Low JGH, Wong WH, Chua YY, Goh SL, Ng HJ. Critically Ill COVID-19 Infected Patients Exhibit Increased Clot Waveform Analysis Parameters Consistent with Hypercoagulability. Am J Hematol 2020. [Epub ahead of print]. doi: 10.1002/ajh.25822.

26. Yang YH, Huang YH, Chuang YH, Peng CM, Wang

Cite this article as: Spagnolo $\mathrm{P}$, Cozzi A, Foà RA, Spinazzola A, Monfardini L, Bnà C, Alì M, Schiaffino S, Sardanelli F. CTderived pulmonary vascular metrics and clinical outcome in COVID-19 patients. Quant Imaging Med Surg 2020;10(6):13251333. doi: 10.21037/qims-20-546
LC, Lin Y'T, Chiang BL. Autoantibodies against human epithelial cells and endothelial cells after severe acute respiratory syndrome (SARS)-associated coronavirus infection. J Med Virol 2005;77:1-7.

27. Tang N, Bai H, Chen X, Gong J, Li D, Sun Z. Anticoagulant treatment is associated with decreased mortality in severe coronavirus disease 2019 patients with coagulopathy. J Thromb Haemost 2020. [Epub ahead of print]. doi: 10.1111/jth.14817.

28. Lodigiani C, Iapichino G, Carenzo L, Cecconi M, Ferrazzi P, Sebastian T, Kucher N, Studt J-D, Sacco C, Alexia B, Sandri MT, Barco S. Venous and arterial thromboembolic complications in COVID-19 patients admitted to an academic hospital in Milan, Italy. Thromb Res 2020. [Epub ahead of print]. doi: 10.1016/j.thromres.2020.04.024.

29. Thachil J, Tang N, Gando S, Falanga A, Cattaneo M, Levi $\mathrm{M}$, Clark $\mathrm{C}$, Iba T. ISTH interim guidance on recognition and management of coagulopathy in COVID-19. J

Thromb Haemost 2020. [Epub ahead of print]. doi: 10.1148/radiol.2020201561.

30. Codari M, Scarabello M, Secchi F, Sforza C, Baselli G, Sardanelli F. Fully automated contour detection of the ascending aorta in cardiac 2D phase-contrast MRI. Magn Reson Imaging 2018;47:77-82. 